Supporting information for

\title{
An ideal one-dimensional antiferromagnetic spin system observed in hydrogen-bonded naphth[2,3-d] imidazol-2-yl nitronyl nitroxide crystal: the role of hydrogen bond
}

Hideaki Nagashima, Hidenari Inoue, and Naoki Yoshioka*

Figure 1S. Variation of the Fermi contact terms and the Mulliken spin densities of the hydrogen atoms with changing the dihedral angles between the aryl rings and the ONCNO moieties (UB3LYP/6$\left.31 \mathrm{G}^{*} / / \mathrm{AM} 1\right)$.

Figure 2S. Angular dependence of calculated $J$ values (O: UB3LYP/4$31 \mathrm{G}$; •: UBLYP/4-31G).

Figure 3S. Angular dependence of calculated $J$ values (O: UB3LYP/431G; •: UBLYP/4-31G). 


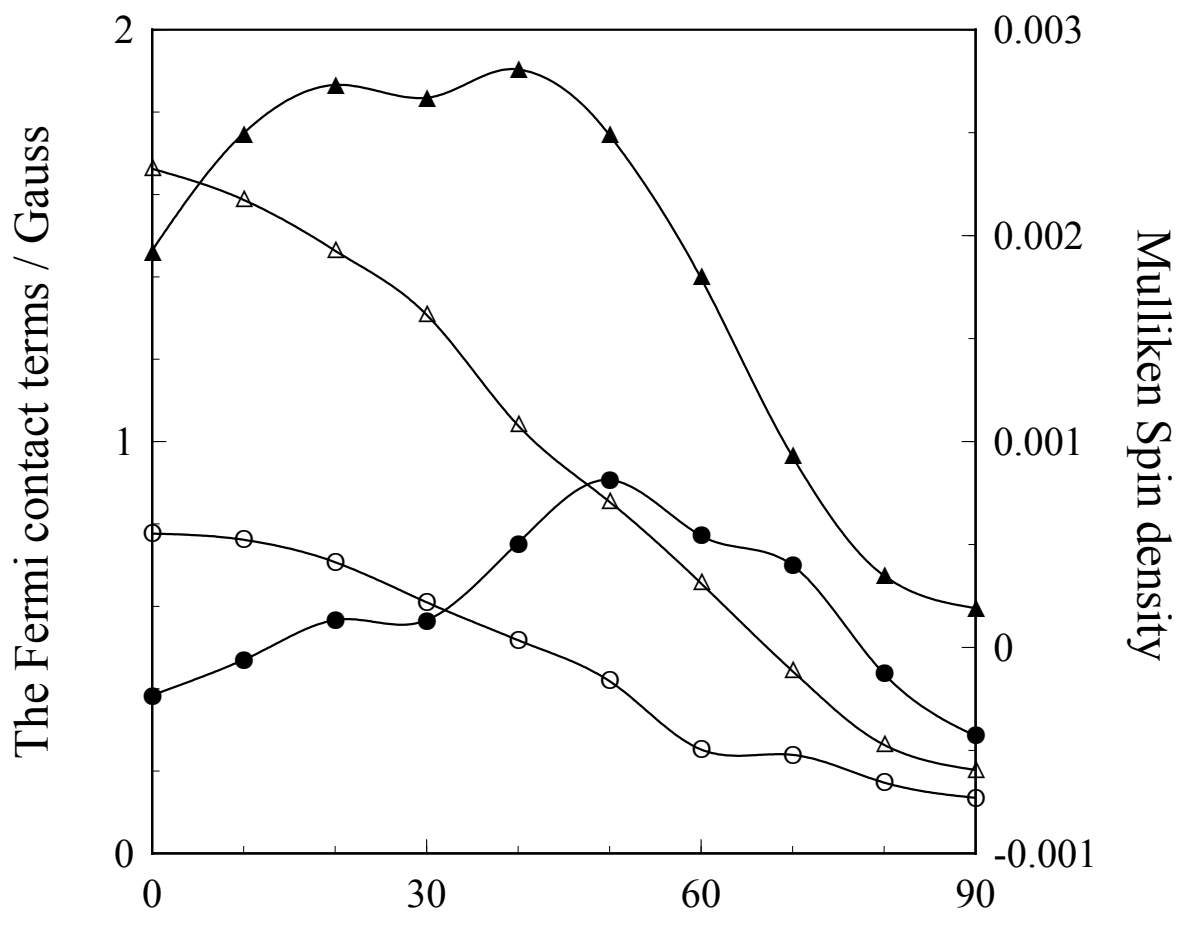

Dihedral angle $/{ }^{\circ}$

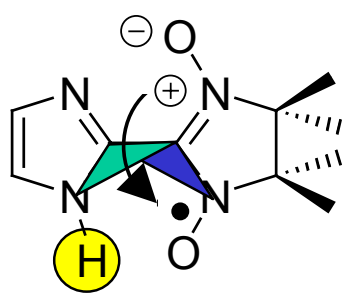

Imidazol-2-yl nitronyl nitroxide (2)

Fermi contact terms $(\mathrm{O})$

Mulliken spin density (O)

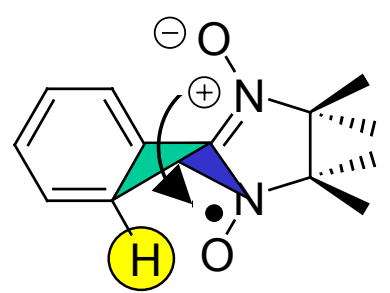

Phenyl nitronyl nitroxide

Fermi contact terms $(\triangle)$ Mulliken spin density $(\mathbf{\Delta})$

Figure 1S. Variation of the Fermi contact terms and the Mulliken spin densities of the hydrogen atoms with changing the dihedral angles between the aryl rings and the ONCNO moieties (UB3LYP/6-31G*//AM1). 


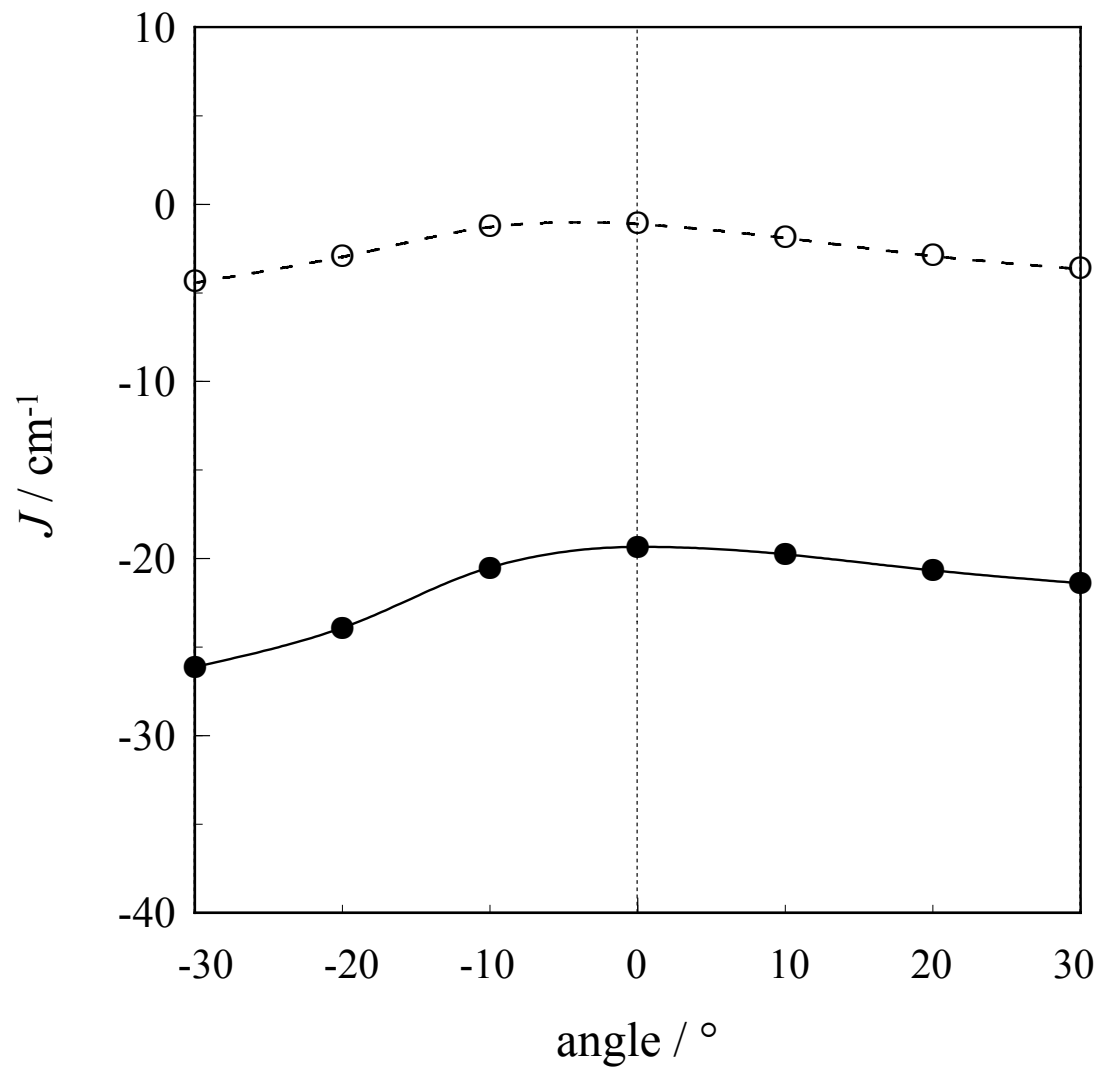

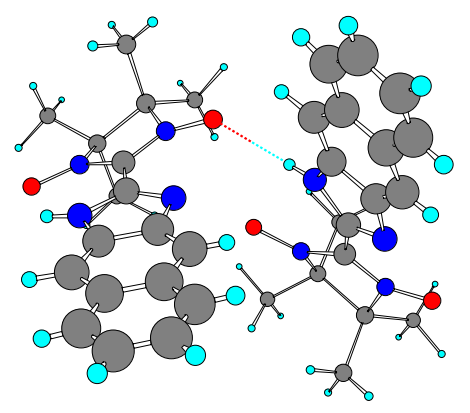

$-30^{\circ}$

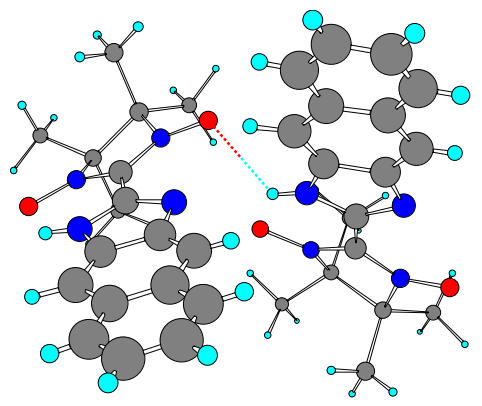

$0^{\circ}$

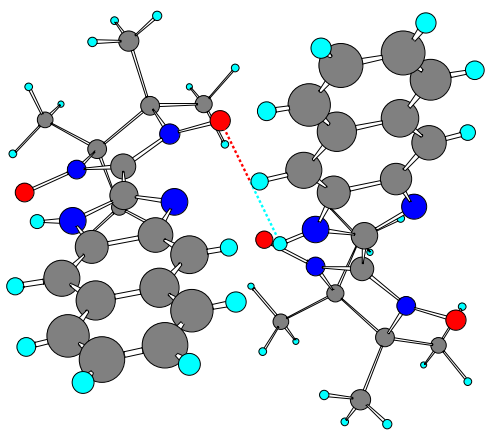

$30^{\circ}$

Figure 2S. Angular dependence of calculated $J$ values (O: UB3LYP/4-31G; $\bullet$ : UBLYP/4$31 \mathrm{G})$. 


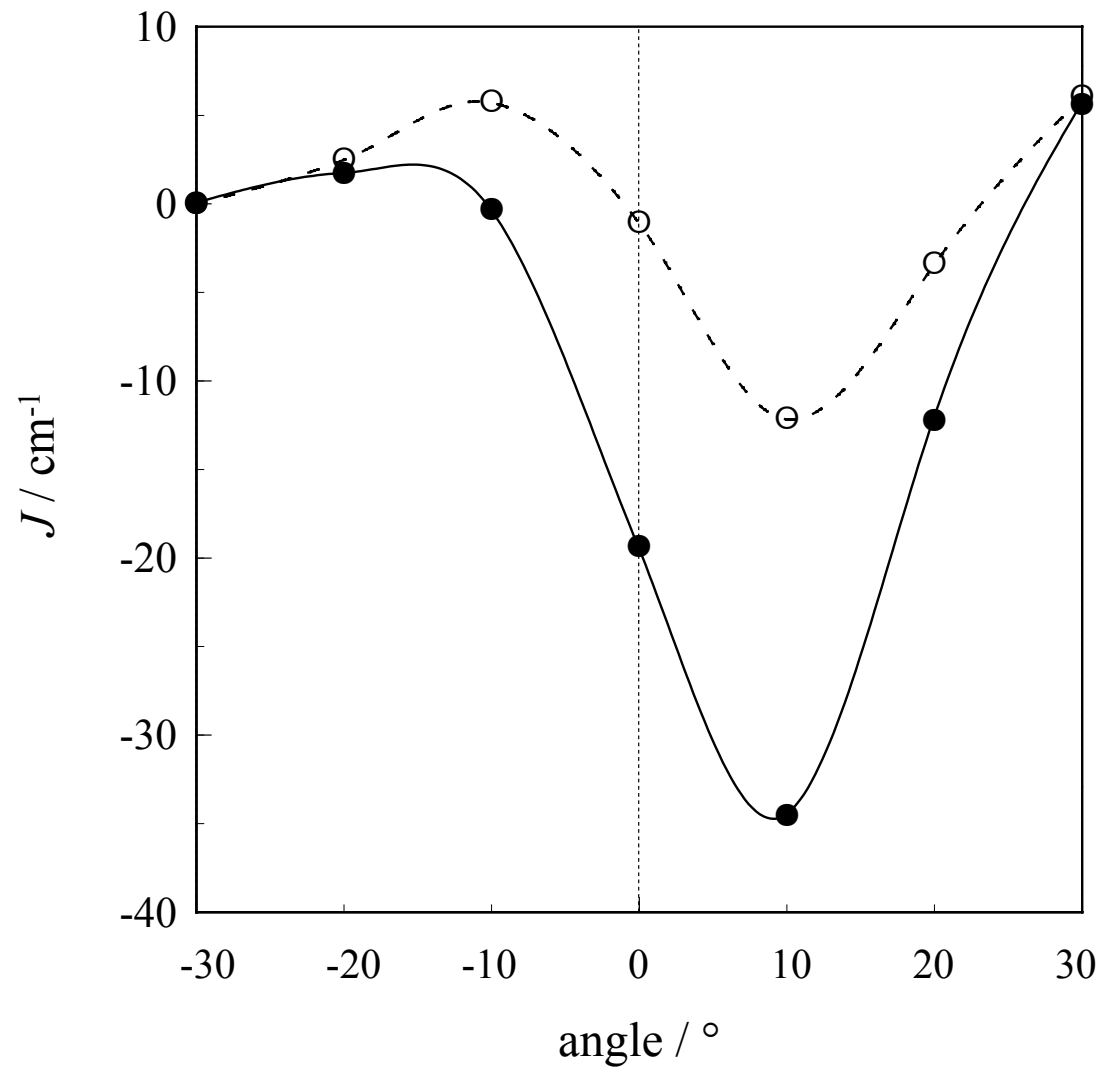

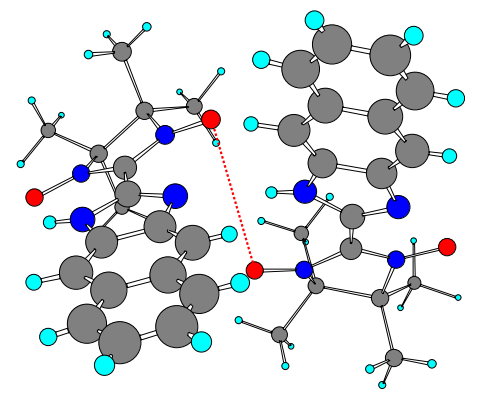

$-30^{\circ}$

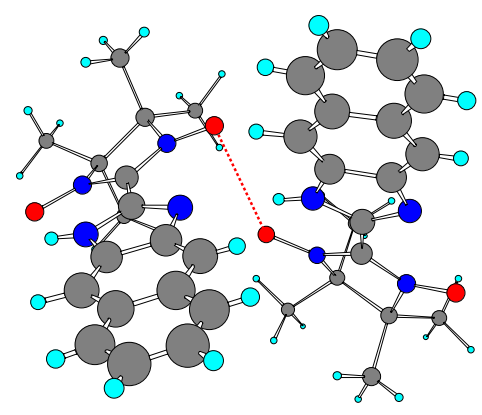

$0^{\circ}$

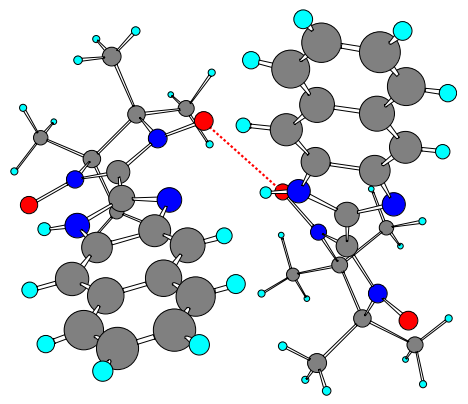

$30^{\circ}$

Figure 3S. Angular dependence of calculated $J$ values (O: UB3LYP/4-31G; •: UBLYP/4$31 \mathrm{G})$. 